\title{
Parâmetros Biofísicos Aplicados no Parque Nacional da Serra das Confusões, Piauí-Brasil
}

\author{
Laurizio Emanuel Ribeiro Alves ${ }^{1}$ (D), Heliofábio Barros Gomes ${ }^{2}$ (D), Felipe Souza dos Santos $^{3}$ (D), \\ Washington Luiz Félix Correia Filho ${ }^{4}$ (D), José Francisco de Oliveira Júnior ${ }^{2}$ \\ ${ }^{\text {I} P r o g r a m a ~ d e ~ P o ́ s-G r a d u a c ̧ a ̃ o ~ e m ~ M e t e o r o l o g i a, ~ I n s t i t u t o ~ N a c i o n a l ~ d e ~ P e s q u i s a s ~ E s p a c i a i s, ~}$ \\ Cachoeira Paulista, SP, Brasil. \\ ${ }^{2}$ Instituto de Ciências Atmosféricas, Universidade Federal de Alagoas, Maceió, AL, Brasil. \\ ${ }^{3}$ Programa de Pós-Graduação em Meteorologia, Instituto de Ciências Atmosféricas, \\ Universidade Federal de Alagoas, Maceió, AL, Brasil. \\ ${ }^{4}$ Programa de Pós-Doutorado, Instituto de Ciências Atmosféricas, \\ Universidade Federal de Alagoas, Maceió, AL, Brasil.
}

Recebido em: 29 de Maio de 2019 - Aceito em: 23 de Abril de 2020

\begin{abstract}
Resumo
O objetivo deste trabalho é avaliar os parâmetros biofísicos, chuva e a temperatura de superfície nas condições da vegetação, no Parque Nacional da Serra das Confusões (PNSC), Piauí-NEB. Com auxílio das imagens obtidas do satélite Landsat 8 foi possível calcular o Normalized Difference Vegetation Index (NDVI) e a temperatura de superfície (Ts). A chuva do PNSC apresentou sazonalidade bem definida, estações seca e chuvosa. Ambas as estações têm papel crucial nas condições da vegetação do PNSC. A classe de NDVI $(>0,693)$ apresentou a maior redução da área de cobertura do PNSC próximo de $72,7 \%$, enquanto que as classes $(0,347-0,433)$ e $(0,433-0,52)$ tiveram um aumento de $34,7 \%$ e $31,8 \%$, respectivamente. Além disso, ficou claro que as condições da vegetação influenciam na $T s$ da região.
\end{abstract}

Palavras-chave: índice de vegetação, temperatura de superfície, sensores orbitais.

\section{Parâmetros Biofísicos Aplicados no Parque Nacional da Serra das Confusões, Piauí-Brasil}

\begin{abstract}
The objective of this work is to evaluate the biophysical parameters, rainfall and surface temperature in the vegetation conditions, in the Serra das Confusões National Park (SCNP), Piau-NEB. With the aid of the images obtained from the satellite Landsat 8 it was possible to calculate the Normalizes Difference Vegetation Index (NDVI) and the surface temperature $(T S)$. The SCNP rainfall presented a well-defined seasonality, dry and rainy seasons, which play a crucial role in the vegetation conditions of the SCNP. The NDVI class $(>0.693)$ had the greatest reduction o the coverage area around $72.7 \%$, while the classes $(0.347-0.433)$ and $(0.433-0.52)$ had an increase of $34.7 \%$ and $31,8 \%$, respectively. In addition, it was clear that vegetation condition influenced the $T s$ of the region.
\end{abstract}

Keywords: vegetation index, surface temperature, orbital sensors.

\section{Introdução}

Os parâmetros biofísicos de superfície são essenciais na avaliação de diferentes ecossistemas e no manejo de áreas extensas (Sakowska et al., 2016). Com essa premissa, foi selecionado o Parque Nacional da Serra das
Confusões (PNSC), no estado do Piauí-Nordeste Brasileiro (NEB), criado pelo Decreto $\mathrm{s} / \mathrm{n}^{\circ}$ de $02.10 .98 \mathrm{com}$ o intuito de preservar o ecossistema natural da região (Gonçalves, 2003). Tal avaliação irá auxiliar no entendimento da dinâmica da vegetação e sua influência na temperatura

Autor de correspondência: Laurizio Emanuel Ribeiro Alves, laurizio.r@gmail.com. 
de superfície do PNSC. O parque é uma região de transição dos biomas Caatinga e Cerrado, com amostras de floresta estacional decidual e savana estépica. $\mathrm{O}$ entendimento da dinâmica desses biomas e sua resposta as variáveis meteorólogicas são essenciais para entendimento do tempo e clima nessa região. Para entender essas variações o monitoramento via Sensoriamento Remoto (SR) viabiliza uma análise rápida e de menor investimento. Vale ressaltar, que a aplicação de índices para o monitoramento e avaliação do uso e ocupação do solo já é consolidado pela comunidade científica (Jensen e Epiphanio, 2011; Sakowska et al., 2016; Freitas et al., 2017), seguido da temperatura de superfície (Coelho e Correa, 2013; Gomes et al., 2017). Neste estudo será usado o índice Normalized Difference Vetation Index (NDVI) proposto por Tucker (1979). Este índice apresenta dois aspectos: o primeiro está relacionado nas mudanças sazonais e interanuais, e o último está ligado a redução de ruídos (sombra de nuvens, por exemplo) e condições da vegetação. Ainda com auxílio do SR é possível obter as condições da temperatura de superfície, que possibilita uma análise espacial da área avaliada (Coelho e Correa, 2013; Gomes et al., 2017).

Baseado no exposto, objetivo é avaliar a chuva e a temperatura de superfície nas condições da vegetação no PNSC, Piauí-NEB.

\section{Materiais e Métodos}

\section{1. Área de estudo}

O PNSC está situado na faixa de contato entre o maciço cristalino da Formação Serra Grande e a Bacia Sedi- mentar do Parnaíba, apresentando duas feições: i) com superfícies planas com altura média de $700 \mathrm{~m}$, formado por amplos vales, e ii) constituída de rochas cristalinas com altitude média de $650 \mathrm{~m}$, com relevo complexo. O PNSC é localizado no estado do Piauí, possui uma área de 526.108 hectares nas coordenadas $9^{\circ} 7^{\prime} 30^{\prime \prime} \mathrm{S}$ de latitude e $43^{\circ} 48^{\prime} 11^{\prime}$ W de longitude Fig. 1. O parque está distribuído nos municípios de Alvorada do Gurguéia, Bom Jesus do Piauí, Brejo do Piauí, Canto do Buriti, Caracol, Cristino Castro, Curimatá, Guaribas, Jurema, Redenção do Gurgueia, Santa Luz e Tamboril do Piauí (Gonçalves, 2003).

O PNSC está situado em uma área de transição caatinga/cerrado com predominância de três tipos de cobertura vegetal: i) Arbórea, ii) Arbustiva e iii) Arbórea/ Arbustiva. Existe a predominância de espécies caducifólias, bem como de sub-bosques de florestas semi-deciduais nas matas ciliares e nas localidades onde as condições edafoclimáticas são mais favoráveis (Gonçalves, 2003).

\subsection{Dados Pluviométricos}

Os dados pluviométricos foram obtidos do Banco de Dados Meteorológicos para Ensino e Pesquisa (BDMEP) (http://www.inmet.gov.br/projetos/rede/pesquisa/). As estações utilizadas foram: Vale do Gurgueia (Cristiano Castro)-PI (OMM: 82870) nas latitude e longitude, $-8,41^{\circ} \mathrm{e}$ $-43,71^{\circ}$ a 265 m; Bom Juses do Piaúi-PI (OMM: 82975) nas latitude longitude, $-9,1^{\circ}$ e $-44,11^{\circ}$ a $331,74 \mathrm{~m}$; e Caracol-PI (OMM: 82976) nas latitude e longitude, $-9,28^{\circ} \mathrm{e}$ $-43,33^{\circ}$ a $522,77 \mathrm{~m}$. Os dados diários pluviométricos correspondem a chuva antecedente a passagem do satélite Landsat 8. O período corresponde ao ano de 2014.

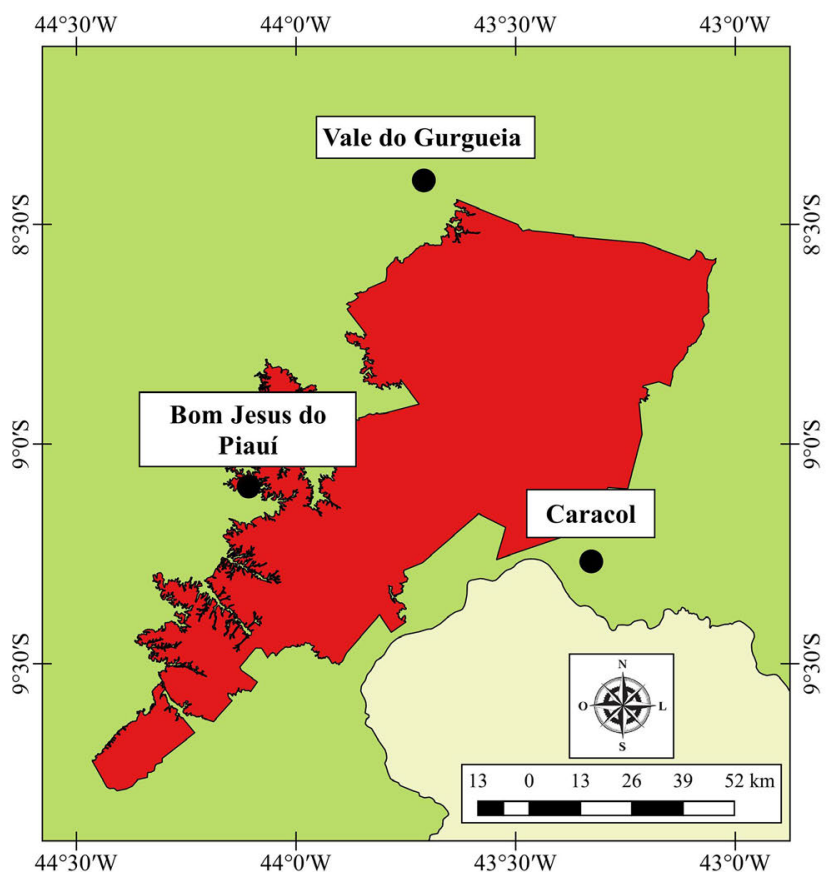

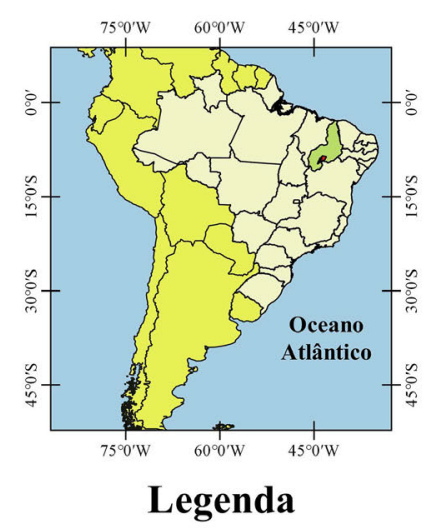

Estações Meteorológicas

Parque Nacional da Serra das Confusões

Piauí

Brasil

América do Sul

Sistema de Coordenadas Geograficas

Latitude / Longitude SIRGAS 2000

Figura 1 - Mapa de localização do Parque Nacional da Serra das Confusões no estado do Piauí, Nordeste Brasileiro e das estações meteorológicas (•). 


\subsection{Imagens de Satélite}

O satélite é o Landsat 8 e possui abordo de sua plataforma os sensores Operational Land Imag (OLI) e o Thermal Infrared Sensor (TIRS). As cenas foram adquiridas pela United State Geological Survey (USGS) via endereço eletrônico: http://earthexplorer.usgs.gov/. As imagens selecionadas apresentam pouca ou nenhuma cobertura de nuvens sobre a área do PNSC. A informações relevantes das imagens selecionadas são apresentadas na Tabela 1.

$\mathrm{O}$ recorte da área de estudo consiste numa forma quadricular onde: canto superior esquerdo $\left(44^{\circ} 06^{\prime} \mathrm{W}\right.$ e $\left.08^{\circ} 24^{\prime} \mathrm{S}\right)$ e canto inferior esquerdo $\left(44^{\circ} 06 \mathrm{~W}\right.$ e $\left.09^{\circ} 18^{\prime} \mathrm{S}\right)$, canto superior direito $\left(42^{\circ} 54^{\prime} \mathrm{W}\right.$ e $\left.08^{\circ} 24^{\prime} \mathrm{S}\right)$ e canto inferior direito $\left(42^{\circ} 54^{\prime} \mathrm{W}\right.$ e $\left.09^{\circ} 18^{\prime} \mathrm{S}\right)$ Fig. 2.

\subsection{Processamento das Imagens}

As imagens foram processadas pelo software QGis versão 2.18 (QGIS DEVELOPMENT TEAM, 2017). Incialmente foi realizado a conversão do número digital $(N D)$ em radiância espectral, segundo a equação proposta por Markham e Baker (1987), (Eq. (1)):

$$
L_{\lambda i}=a_{i}+\frac{b_{i}-a_{i}}{255} * N D
$$

em que, $a$ e $b$ são as radiâncias espectrais mínima e máxima (W.m ${ }^{-2} \cdot \mathrm{sr}^{-1} \cdot \mathrm{Mm}^{-1}$ ), detectadas pelo OLI e TIRS do satélite Landsat; $i$ corresponde às bandas 4 e 5 do sensor OLI, e banda 10 do sensor TIRS do Landsat 8, conforme (Tabela 2).

Tabela 1 - Informações das imagens dos sensores OLI/TIRS do satélite Landsat 8, seguido do horário da passagem, ponto/órbita, ângulo zenital e distância Terra-Sol.

\begin{tabular}{lccc}
\hline Data & Horário da Passagem (UTC) & Ponto/órbita & Ângulo Zenital $\left({ }^{\circ}\right)$ \\
\hline $15 / 05 / 2014$ & $12: 59$ & $219 / 66$ & 51.40 \\
$16 / 06 / 2014$ & $13: 00$ & $219 / 66$ & 47.65 \\
$02 / 07 / 2014$ & $13: 00$ & $219 / 66$ & 47.36 \\
$03 / 08 / 2014$ & $13: 00$ & $219 / 66$ & 50.71 \\
$04 / 09 / 2014$ & $13: 00$ & $219 / 66$ & 58.07 \\
\hline
\end{tabular}

Fonte: United State Geological Survey (2018).

*UA-Unidade Astronômica.

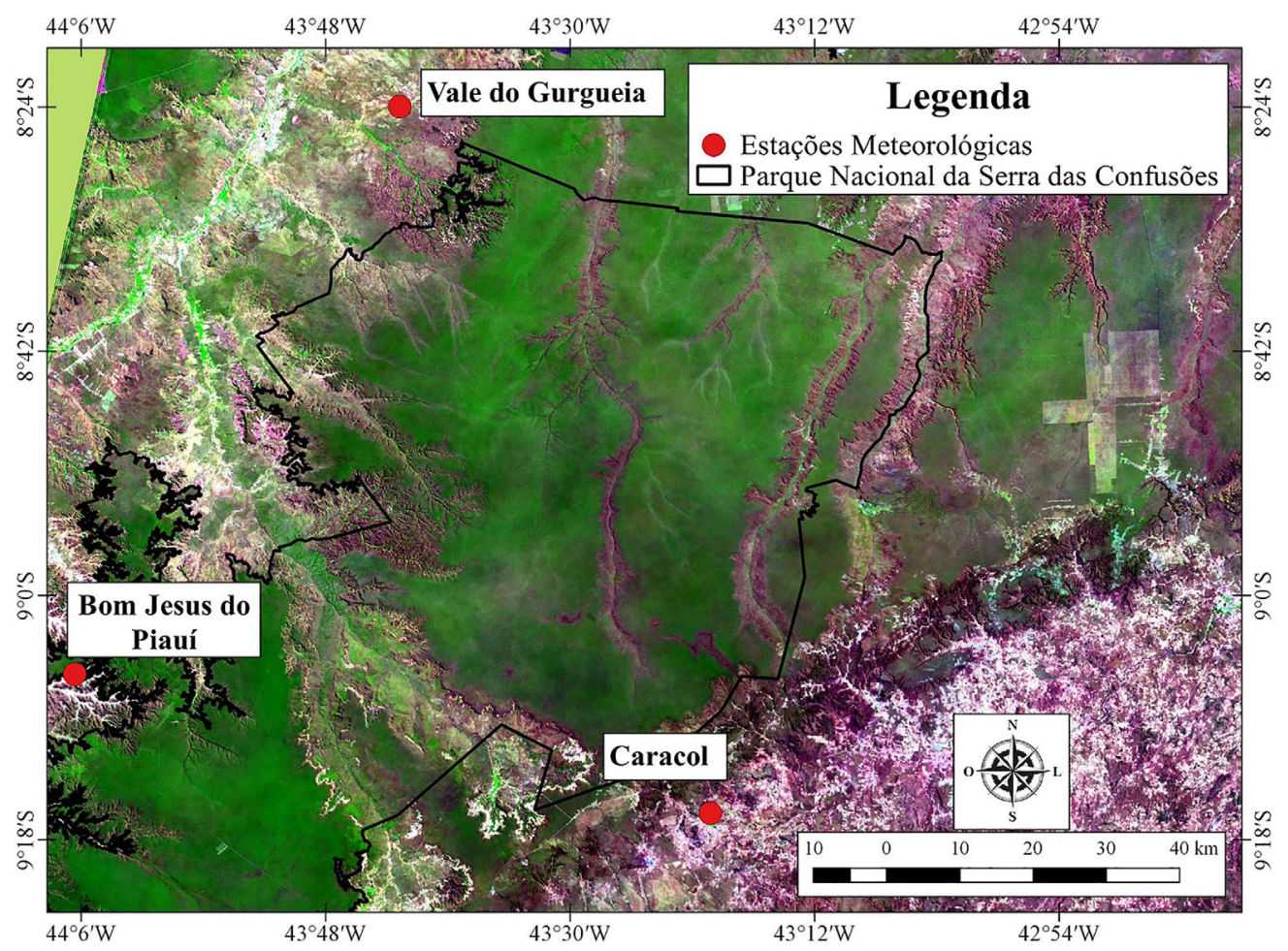

Figura 2 - Recorte da imagem OLI-Landsat 8 da área de estudo com combinação RGB das bandas espectrais 5, 4, 3 em composição falsa cor para o dia 04/09/2014, realçando as estações meteorológicas. 
Tabela 2 - Descrição das bandas do OLI e TIRS do Landsat8, com os respectivos coeficientes de calibração $\left(a_{i}\right.$ e $\left.b_{i}\right)$ e irradiação solar espectral $\left(E_{\lambda i}\right)$.

\begin{tabular}{lcccc}
\hline Descrição das bandas & Comprimento de onda & \multicolumn{2}{c}{ Coeficiente de calibração } \\
\cline { 3 - 5 } TIRS/OLI & & $a_{i}$ & $b_{i}$ \\
\hline Banda 4 (Vermelho; OLI) & $0,64-0,67$ & $-48,32$ & 585,11 & 1562,8 \\
Banda 5 (Infravermelho Próximo; OLI) & $0,85-0,88$ & $-29,57$ & 358,06 & 956,4 \\
Banda 10 (Infravermelho termal: TIRS) & $10,60-11,19$ & 0,10 & 22,00 \\
\hline
\end{tabular}

Fonte: Barsi et al. (2014); Silva et al. (2016),

Em seguida foi calculado a reflectância monocromática de cada banda ( $\rho \lambda i)$, definida como a razão entre o fluxo de radiação refletido e o fluxo de radiação solar incidente, foi obtida segundo a Eq. (2) (Allen et al., 2002):

$$
\rho_{\lambda i}=\frac{\pi * L_{\lambda i}}{E_{\lambda i} * \cos Z * d_{r}}
$$

em que $E_{\lambda i}$ é a irradiância solar espectral de cada banda no topo da atmosfera $\left(\mathrm{W} \cdot \mathrm{m}^{-2} \cdot \mathrm{sr}^{-1} \cdot \mu \mathrm{m}^{-1}\right)$, a qual é apresentada na Tabela $2, Z\left(^{\circ}\right)$ é o ângulo zenital solar e $d_{r}(U A)$ é a distância Terra-Sol (Tabela 1).

Após o cômputo da $\rho \lambda i$ foi calculado o $N D V I$, o qual é a razão entre as bandas espectrais do infravermelho próximo e do vermelho e a soma das mesmas reflectâncias, conforme a Eq. (3), proposto por Tucker (1979):

$$
N D V I=\frac{I V-V}{I V+V}
$$

em que, $I V$ é a banda do espectro do infravermelho-próximo e $V$ é a banda do espectro do vermelho. O NDVI é um indicador das condições da vegetação e varia entre -1 a +1 . Geralmente valores negativos são atribuídos a corpos d'água, valores próximos de zero corresponde a áreas sem cobertura vegetal, já valores próximos de +1 é atribuído a áreas densamente vegetadas (Freitas et al., 2017).

A temperatura de superfície $(T S)$ foi calculada com base na Eq. (4), proposta por Allen et al. (2002):

$$
T s=\frac{K_{2}}{\ln \left(\frac{\varepsilon * K_{1}}{L_{\lambda 10}}+1\right)}
$$

em que $K_{1}=774,89 \mathrm{Wm}^{-2} \mathrm{sr}^{-1} \mu \mathrm{m}^{-1}$ e $K_{2}=1321,08 \mathrm{~K}$ são constantes de calibração da banda termal do Landsat8; $e$ é a emissividade termal, a qual segundo Allen et al. (2002), ela pode ser obtida quando o $N D V I>0$ e $I A F<3$, e é calculado de acordo com a (Eq. (5)):

$$
\varepsilon=0,97+0,00331 * I A F
$$

onde o SAVI (Soil Vegetation Index) proposto por Huete (1988) é calculado segundo a (Eq. (7)):

$$
S A V I=\frac{(1+L)(I V-V)}{L+I V+V}
$$

em que $L$ é o coeficiente de ajusto do solo, para este estudo foi adotado 0,5 , pois a vegetação do PNSC foi considerada com densidade de vegetação intermediária, conforme Huete (1988).

\section{Resultados e Discussão}

Na Fig. 3 são apresentados os totais pluviométricos das três estações meteorológicas utilizadas no estudo. Claramente, na região de estudo existem dois regimes pluviométricos bem definidos, uma estação chuvosa correspondente a seis meses chuvosos (novembro a abril) e uma estação seca com seis meses secos (maio a outubro). A atuação de Vórtices Ciclônicos de Altos Níveis (VCAN) são um dos principais, senão o principal, responsáveis pelo regime pluviométrico no parque que pode sofrer também influências, porém em menor escala, da Zona de Convergência Intertropical (ZCIT) e resquícios de frentes que se desprendem da Zona de Convergência do Atlântico Sul (ZCAS) (Correia Filho et al., 2019; Fedorova et al., 2017; Kousky e Gan, 1981). Além disso, os maiores registros pluviométricos foram observados nas estações de Vale do Gurgueia (196,4 mm) e Bons Jesus do Piauí $(239,8 \mathrm{~mm})$ no mês de março, respectivamente. A exceção, a estação de Caracol com 185,4 mm no mês de abril. Nos meses correspondentes a estação seca não houve registro de chuva. Destaque para a estação do Vale do Gurgueia com 159 dias sem chuva.

Os resultados obtidos corroboram com a avaliação do Atlas Climatológico do Estado Piauí (Andrade Júnior et al., 2004). Moscati e Gan (2006) mostraram que no Piauí, o mês mais seco corresponde a setembro e o mais chuvoso é março. Além disso, eles destacam que tal variação está associada ao deslocamento da Zona de Convergência Intertropical (ZCIT), que é um dos mais importantes sistemas meteorológicos produtores de chuva no Nordeste (Uvo, 1989; Lyra et al., 2014).

As condições da vegetação do PNSC entre os meses de maio a setembro de 2014 são apresentadas na Fig. 4. Na análise espacial os valores obtidos do NDVI foram diminuindo significativamente ao longo do tempo. Esse fato está ligado diretamente ao regime pluviométrico da região, 
Vale do Gurgueia (Cristiano Castro) - PI (OMM: 82870 )

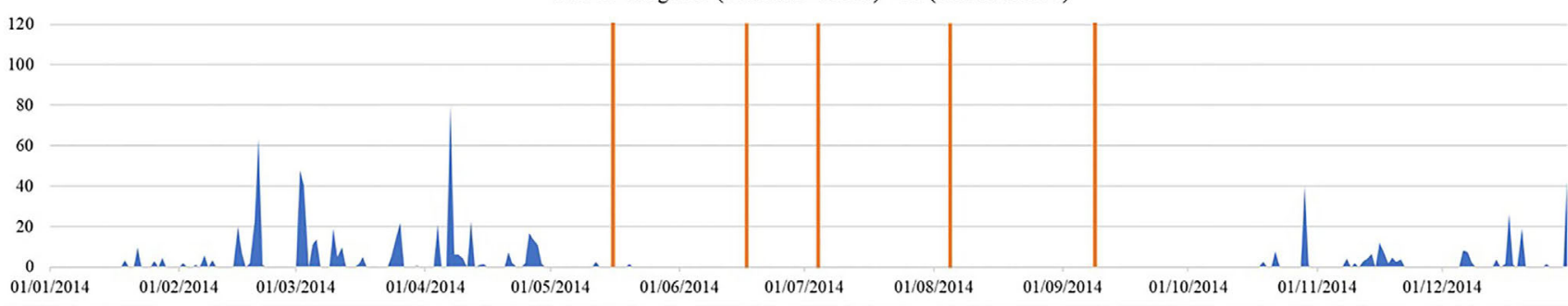

Bom Jesus do Piauí - PI (OMM: 82975)

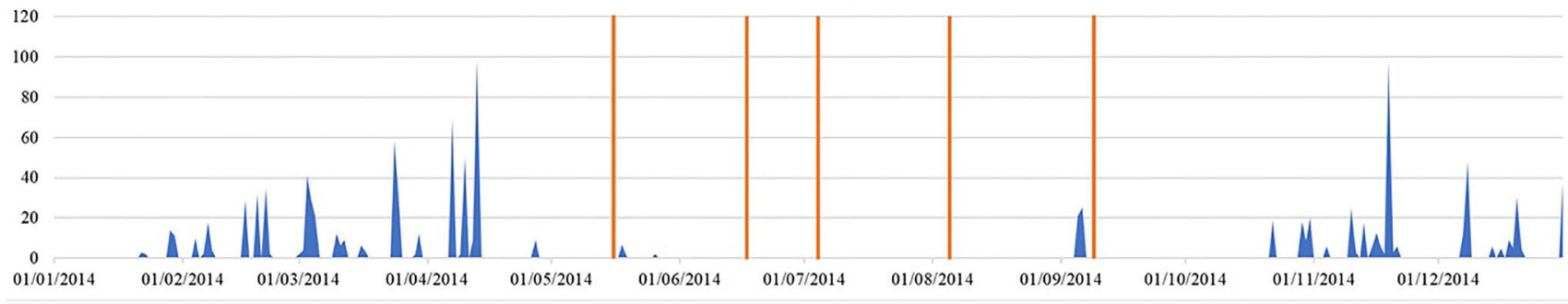

Caracol - PI (OMM: 82976)

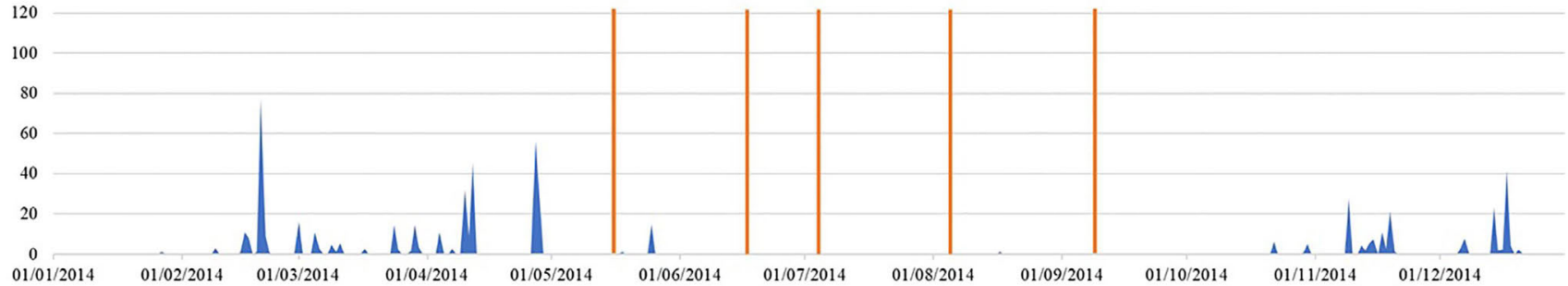

Figura 3 - Total pluviométrico (mm) diário para o período de 01 de maio de 2014 a 31 de outubro de 2014 nas estações meteorológicas, seguido da indicação das datas das imagens, respectivamente.
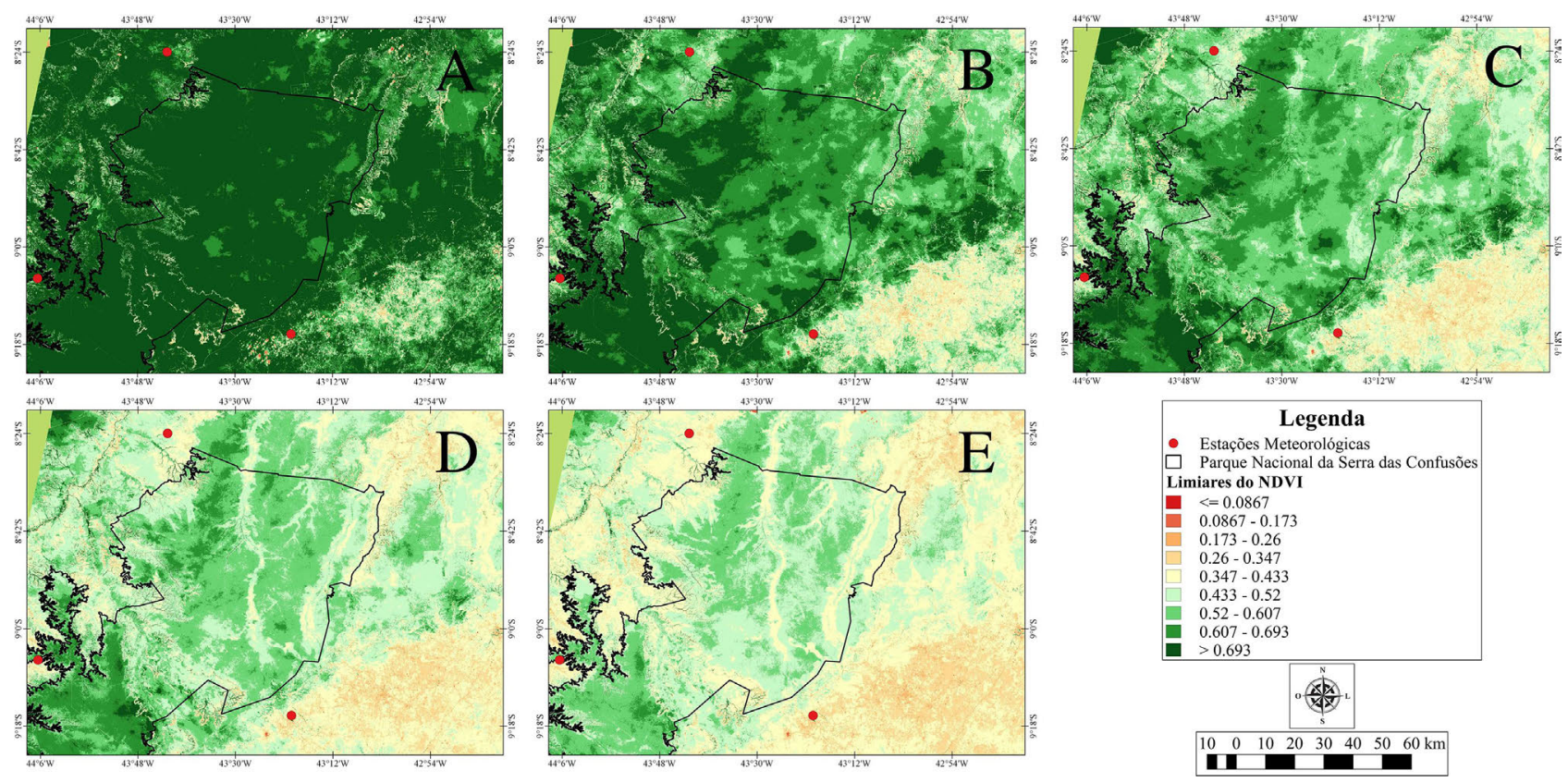

Figura 4 - Mapa temporal da variação do NDVI do Parque Nacional da Serra das Confusões e áreas adjacentes para os dias 15/05/2014 (A), 16/06/2014 (B), 02/07/2014 (C), 03/08/2014 (D) e 04/09/2014 (E) 
pois os meses antecedentes a maio apresentaram os maiores registros pluviométricos, enquanto que os meses subsequentes praticamente não possuem registros de chuva Fig. 3. Além disso, é sabido que os biomas da Caatinga e do Cerrado apresentam forte dependência da chuva para manter o seu vigor, principalmente a Caatinga (Trovão et al., 2007).

A redução do vigor da vegetação associado à chuva é notória no PNSC. Inicialmente em 15/05/2014 (Fig. 4a) os valores obtidos de NDVI foram altos, com uma área de $13.731,4 \mathrm{~km}^{2}$ e superiores a 0,693 . À medida que o período de estiagem se estabelece na região, os valores de NDVI vão reduzindo significativamente, principalmente em 04/09/2014 com uma área menor $\left(43,8 \mathrm{~km}^{2}\right.$ ) (Fig. 4e). É evidente a influência marcante da chuva nas condições da vegetação do PNSC, visto que o mês de maio (estação chuvosa) apresenta os maiores valores de NDVI, seguido de setembro, o qual é considerado o mês mais seco (Moscati e Gan, 2006), que por sua vez apresentou os menores valores de NDVI.

A cronologia das variações da área de cobertura do PNSC em relação as cinco classes (0,347-0,433 (vegetação rasteira); 0,433-0,52 (vegetação rasteira/arbustiva); 0,52-0,607 (vegetação arbustiva); 0,607-0,693 (vegetação arbórea); >0,693 ( vegetação arbórea densa)) apresentaram maior variação na área de cobertura do NDVI no período de maio a setembro de 2014 Fig. 5. Há uma redução das maiores classes (0,607-0,693 e >0,693) de NDVI, que por sua vez coincidem com áreas de vegetação com maior vigor, principalmente a maior classe $(>0,693)$ que apresentou atenuação na área de cobertura, na ordem de $13.687,6 \mathrm{~km}^{2}(72,7 \%)$. Vale destacar que apenas a classe $>$ 0,693 apresentou valor discrepante (outlier) em relação as demais classes. Em contrapartida, as menores classes apresentam um aumento em sua área de cobertura, sobretudo a classe (0.347-0.433) com aumento a área em $6.544,9 \mathrm{~km}^{2}(34,7 \%)$, seguido da classe $(0,433-0,52)$ com $5.982,4 \mathrm{~km}^{2}(31,8 \%)$ e $(0.52-0.607)$ com $2126,5 \mathrm{~km}^{2}$ $(11,3 \%)$.

Resultados similares são encontrados na literatura, por exemplo, Freitas et al. (2017) avaliaram as condições da Caatinga do município de Remanso-BA. Eles notaram que a flora local apresentou alta dependência com o regime pluviométrico da região. Os maiores valores de NDVI no período chuvoso e menores quando no período de escassez de chuva. Becerra, Shimabukuro e Alvalá (2009) avaliaram o padrão sazonal de fisionomias do Cerrado no Estado de Tocantins e constataram que as condições da vegetação seguem os padrões de chuva, e destaca que área de floresta não apresentaram variações acentuadas nos valores de NDVI, apenas um decréscimo no mês de setembro de 2004.

A (Tabela 3) apresenta os valores de máxima, média, mínima e desvio padrão da $T s$ para as imagens selecionadas no estudo. $\mathrm{O}$ menor valor de $T s\left(11,5^{\circ} \mathrm{C}\right)$ foi obtido em

Tabela 3 - Valores de máxima, média, mínima e desvio padrão da $T s\left({ }^{\circ} \mathrm{C}\right)$ para os dias estudados.

\begin{tabular}{lcccc}
\hline Data & Máxima & Média & Mínima & Desvio padrão \\
\hline $15 / 05 / 2014$ & 33.7 & 25.1 & 15.2 & 1.53 \\
$16 / 06 / 2014$ & 35.6 & 25.8 & 17.9 & 2.91 \\
$02 / 07 / 2014$ & 37.9 & 28.4 & 22.6 & 2.09 \\
$03 / 08 / 2014$ & 40.5 & 30.2 & 11.5 & 2.18 \\
$04 / 09 / 2014$ & 42.4 & 33.5 & 13.6 & 2.25 \\
\hline
\end{tabular}

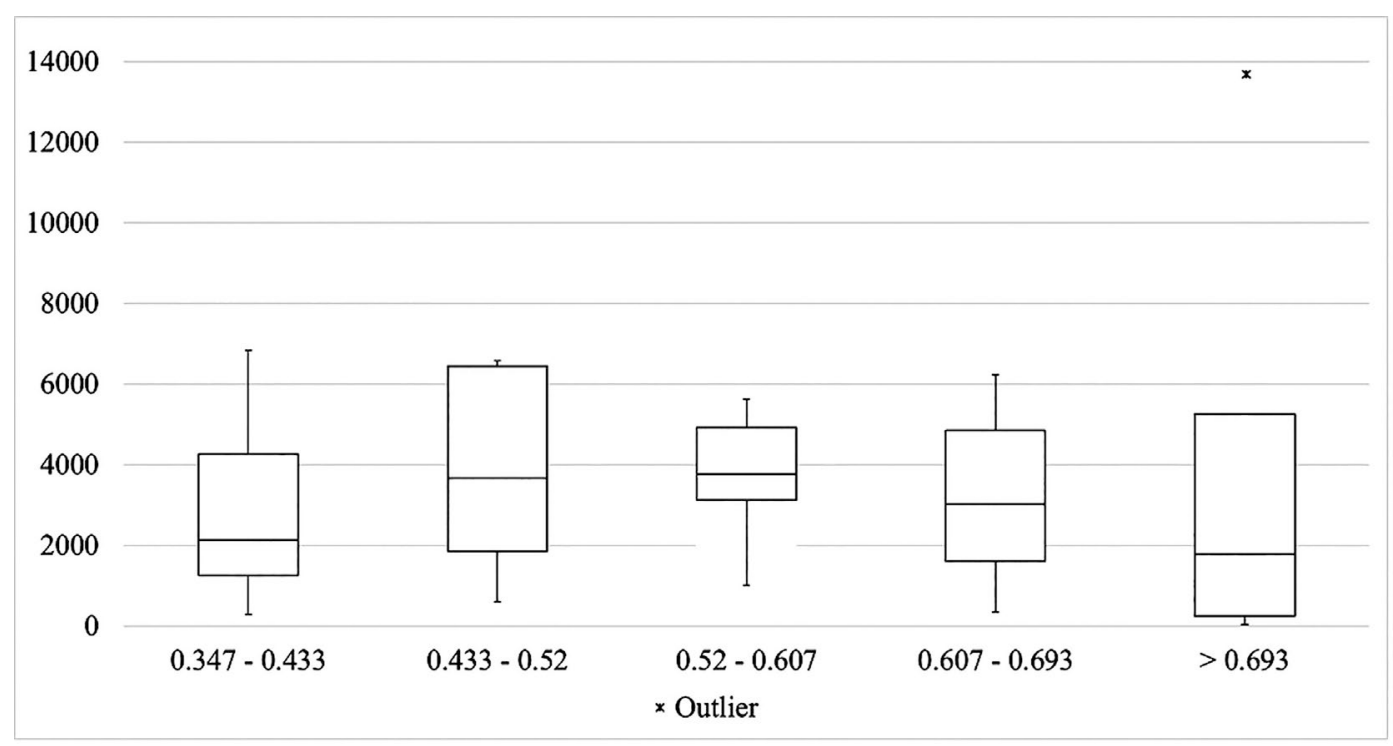

Figura 5 - Variação da área de cobertura do NDVI das cinco classes $(0,347-0,433 ; 0,433-0,52 ; 0,52-0,607 ; 0,607-0,693 ;>0,693)$ para os meses de maio, junho, julho, agosto e setembro de 2014. 

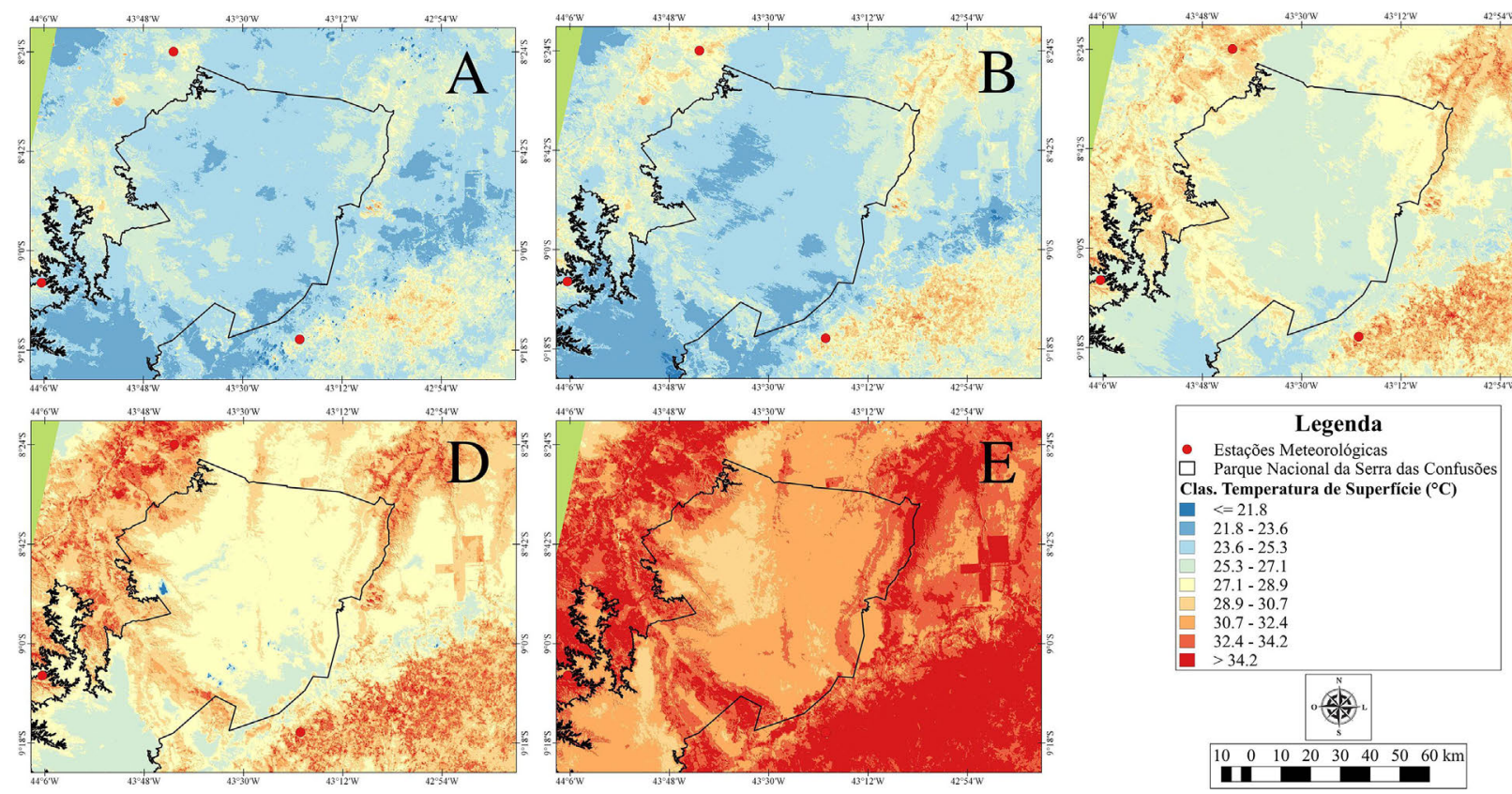

Figura 6 - Mapa temporal da variação da $T s\left({ }^{\circ} \mathrm{C}\right)$ do Parque Nacional da Serra das Confusões e áreas adjacentes para os dias 15/05/2014 (A), 16/06/2014 (B), 02/07/2014 (C), 03/08/2014 (D) e 04/09/2014 (E).

03/08/2014 e o maior valor de $T s\left(42,4{ }^{\circ} \mathrm{C}\right)$ em $04 / 09 /$ 2014. A Ts média da PNSC apresentou seu menor valor em 15/05/2014 e maior em 04/09/2014. Esse comportamento se deve a escassez de chuva, após o mês de maio, que contribuiu para que na região permanecesse seca e quente. De acordo com Andrade Júnior et al. (2004), os meses de maio, junho e julho apresentam temperatura média variando entre $26{ }^{\circ} \mathrm{C}$ a $28{ }^{\circ} \mathrm{C}$, agosto varia entre $26{ }^{\circ} \mathrm{C}$ a $30^{\circ} \mathrm{C}$ e setembro entre $26^{\circ} \mathrm{C}$ a $32^{\circ} \mathrm{C}$. Nessa perspectiva, a temperatura entre os meses de maio a julho de 2014 fora inferior à média climatológica, enquanto que agosto e setembro foram ligeiramente superiores a essa média. O dia 16 de junho de 2014 apresenta o maior valor de desvio padrão $(2,91)$, consequentemente, apresenta a maior dispersão dos valores em tono da média, ao passo que a menor dispersão ocorre no dia 15 de maio de 2014 $(1,53)$.

A variação temporal da $T s$ é mostrada na Fig. 6. Verifica-se um aumento da $T s$, principalmente nas áreas adjacentes aos limites do PNSC, que possui menores valores de NDVI. Enquanto as áreas limítrofes do parque apresentam valores maiores de NDVI e, consequentemente, menores valores de $T s$. Este comportamento também é constatado em outros estudos, tais como: Ferreira et al. (2012), avaliaram a dinâmica da vegetação de Caatinga no município de Petrolina-PE. Eles verificaram que áreas com maiores (menores) valores de NDVI apresentam menores (maiores) valores de Ts. Fausto et al. (2016), também encontraram resultados similares ao deste estudo quando avaliaram as condições da vegetação e da $T s$ em áreas de Cerrado no sul do Estado do Mato Grosso.
Ressalta-se que ausência de chuva por um longo período na região também contribuiu para o aumento acentuado da temperatura no PNSC.

\section{Considerações Finais}

Ao longo do período do El Niño 2015/2016, foram registrados 53 episódios de ressaca na área ALFA (A) e 39 O regime sazonal pluviométrico no Parque Nacional da Serra das Confusões no ano de 2014 apresenta similar a climatologia da região, com estações bem definidas (seca e chuvosa). Os valores de NDVI estimados via sensor orbital (OLI) do satélite Landsat 8 , mostra de forma clara a influência da chuva nas condições da vegetação do Parque Nacional da Serra das Confusões. A variabilidade é marcante, sobretudo na maior classe de NDVI $(>0,693)$, uma vez que inicialmente no mês de maio obteve maior área de cobertura em comparação as demais classes à medida que a chuva diminui na região ocorrem uma redução na área de cobertura em setembro essa área é de $43,8 \mathrm{~km}^{2}$, que representa uma redução em torno de $72,7 \%$ dessa classe. As menores classes de NDVI se sobressaem com um aumento na área de cobertura, correspondente a perda do vigor e saúde da vegetação no Parque Nacional da Serra das Confusões.

Além do mais, a partir da avaliação espacial dos valores de NDVI e $T s$ mostram que são inversos no Parque Nacional da Serra das Confusões. O NDVI diminuí seus valores entre maio e setembro, ao passo que a $T S$ aumenta. 


\section{Referências}

ALLEN, R.G.; TASUMI, M.; TREZZA, R.; WATERS, R.; BASTIAANSSEN, W. et al. Surface Energy Balance Algorithms for Land (SEBAL). Idaho ImplementationAdvanced Training and User's Manual, v. 1.0, p. 97, 2002.

ANDRADE JÚNIOR, A.S.; BASTOS, E.A.; DA SILVA, C.O.; GOMES, A.A.N.; FIGUEREDO JÚNIOR, L.G.M. et al. G. M. Atlas Climatológico do Estado do Piauí. Embrapa Meio-Norte-Documentos (INFOTECA-E), 2004.

BARSI, J.A.; LEE, K.; KVARAN, G.; MARKHAM, B.L.; PEDELTY, J.A. et al. The spectral Response of the Landsat-8 Operational Land Imager. Remote Sensing, v. 6, n. 10, p. 10232-10251, 2014.

BECERRA, J.A.B.; SHIMABUKURO, Y.E.; ALVALÁ, R.C.S. Relação do padrão sazonal da vegetação com a precipitação na região de Cerrado da Amazônia LEGAL, usando índices espectrais de vegetação. Revista Brasileira de Meteorologia, v. 24, n. 2, p. 125-134, 2009.

COELHO, A.L.N.; CORREA, W.S.C. Temperatura de superfície celsius do sensor TIRS/Landsat-8: Metodologia e aplicações. Revista Geográfica Acadêmica, v. 7, n. 1, p. 31-45, 2013.

CORREIA FILHO, W.L.F.; DE OLIVEIRA-JÚNIOR, J.F.; DE BARROS SANTIAGO, D.; DE BODAS TERASSI, P.M.; TEODORO, P.E.; DE GOIS, G.; BLANCO, C.J.C.; SOUZA, P.H.A.; COSTA, M.S.; GOMES, H.B.; SANTOS, P.J. et al. Rainfall variability in the Brazilian northeast biomes and their interactions with meteorological systems and ENSO via CHELSA product. Big Earth Data, v. 3, n. 4, p. 315-337, 2019.

FAUSTO, M.A.; ANGELINI, L.P.; MARQUES, H.O.; MACHADO, N.G.; BIUDES, M.S. et al. Impacto da alteração do uso do solo no saldo de radiação no Cerrado do sul de Mato Grosso. Ambiente \& Água. An Interdisciplinary Journal of Applied Science, v. 11, n. 2, p.350-361, 2016.

FEDOROVA, N.; DOS SANTOS, D.M.B.; SEGUNDO, M.M. L.; LEVIT, V. Middle tropospheric cyclonic vortex in Northeastern Brazil and the Tropical Atlantic. Pure and Applied Geophysics, v. 174, n. 1, p. 397-411, 2017.

FERREIRA, J.M.S.; DOS SANTOS FERREIRA, H.; DA SILVA, H.A.; DOS SANTOS, A.M.; GALVÍNCIO, J.D. et al. Análise espaço-temporal da dinâmica da vegetação de Caatinga no município de Petrolina-PE. Revista Brasileira de Geografia Física, v. 5, n. 4, p. 904-922, 2012.

FREITAS, I.G.; ALVES, L.E.; GOMES, H.B.; JÚNIOR, J.R.; SANTIAGO, D.B.; SILVA, R.A. et al. A. analysis of behavior of vegetation in the year of 2016 for the municipality of Remanso-BA. Revista Geama, v. 3, n. 3, p. 149-156. 2017.
GOMES, H.B.; CAVALCANTE, L.B.; SILVA JUNIOR, R.S.D.; SANTOS, M.N.D. et al. Temperatura da superfície e albedo na região de Ilha Solteira, São Paulo. Mercator, v. 16, e16018, 2017.

GONÇALVES, M.R. Plano de Manejo do Parque Nacional da Serra das Confusões-PI. Brasília, IBAMA. p. 230, 2003.

JENSEN, J.R.; EPIPHANO, J.C.N. Sensoriamento Remoto do Ambiente: Uma Perspectiva em Recursos Terrestres. São Paulo: Parêntese, 2011.

KOUSKY, V.E.; ALONSO GAN, M. Upper tropospheric cyclonic vortices in the tropical South Atlantic. Tellus, v. 33, n. 6, p. 538-551, 1981.

LYRA, G.B.; OLIVEIRA JÚNIOR, J.F.; ZERI, M. Cluster analysis applied to the spatial and temporal variability of monthly rainfall in Alagoas state, Northeast of Brazil. International Journal of Climatology, v. 34, n. 13, p. 3546-3558, 2014.

MOLION, L.C.B.; BERNARDO, S.O. Uma revisão da dinâmica das chuvas no Nordeste brasileiro. Revista Brasileira de Meteorologia, v. 17, n. 1, p. 1-10, 2002.

QUANTUM, G.I.S. Development Team. Quantum GIS Geographic Information System. Open Source Geospatial Foundation Project. 2013.

SAKOWSKA, K.; JUSZCZAK, R.; GIANELLE, D. Remote sensing of grassland biophysical parameters in the context of the Sentinel-2 Satellite mission. Journal of Sensors, v. 2016, e4612809, 2016.

SILVA, B.B.D.; BRAGA, A.C.; BRAGA, C.C.; de OLIVEIRA, L.M.; MONTENEGRO, S.M.; BARBOSA JUNIOR, B. et al. Procedures for calculation of the albedo with OLILandsat 8 images: Application to the Brazilian semi-arid. Revista Brasileira de Engenharia Agrícola e Ambiental, v. 20, n. 1, p. 3-8, 2016.

TROVÃO, D.M.D.; FERNANDES, P.D.; ANDRADE, L.A.D.; DANTAS NETO, J. et al. Variações sazonais de aspectos fisiológicos de espécies da Caatinga. Revista Braileira de Engenharia Agrícola e Ambiental, v. 11, n. 3, p. 307-311, 2007.

TUCKER, C.J. Red and photographic infrared linear combinations for monitoring vegetation. Remote Sensing of Environment, v. 8, p. 127-150, 1979.

UVO, C.R.B. A Zona de Convergência Intertropical (ZCIT) e sua Relação com a Precipitação da Região Norte do Nordeste Brasileiro. Dissertação de Mestrado em Meteorologia, Instituto Nacional de Pesquisas Espaciais, 1989.

License information: This is an open-access article distributed under the terms of the Creative Commons Attribution License (type CC-BY), which permits unrestricted use, distribution and reproduction in any medium, provided the original article is properly cited. 